\title{
Bruits associés aux fonctionnements de diverses turbomachines
}

\author{
Noise associated with the operation \\ of various turbomachines
}

\author{
P. Boussuges, S. Casacci, M. Wegner \\ Société Neyrpic, Grenoble
}

\section{Introduction}

Les constructeurs de turbomachines hydrauliques sont concernés par les phénomènes de bruit à deux titres :

- la mesure du bruit peut représenter une méthode élégante de diagnostic et de contrôle des phénomènes hydrodynamiques instationnaires;

- les cahiers des charges requièrent des niveaux sonores à respecter du point de vue de la gêne humaine; la responsabilité du constructeur est donc à priori engagée, même si dans l'immédiat délimiter scientifiquement l'intensité vraie du bruit émis par la machine pose problème.

Considérant qu'il est prématuré d'édicter des lois générales en matière de mesure des niveaux sonores autour des turbomachines, aussi bien que de corréler rigidement ces niveaux avec les divers types de machines et/ou de régime de fonctionnement, cette contribution essaiera de présenter un échantillon varié de résultats de mesures sur modèle et sur prototype. Cet échantillon permettra de tirer trois enseignements, de caractère qualitatif pour l'instant, à savoir :

- la plupart des évolutions du niveau sonore en fonction du régime de fonctionnement paraissent relativement logiques du point de vue hydrodynamique ;

- le niveau de bruit varie considérablement en fonction de l'emplacement de l'observateur au voisinage de la turbomachine;

- la séparation entre le niveau sonore émis par la source "turbomachine" et les niveaux sonores émis par les sources "parasites" à savoir alternateur, pompes auxiliaires, etc., n'étant pas réalisée, toute interprétation des mesures de bruit reste fortement approximative. Un travail technique considérable reste donc à exécuter pour passer à un stade scientifique.
Niveaux sonores sur un modèle réduit de turbine Francis

1) Le modèle se caractérise par les paramètres utiles suivants :

vitesse spécifique $n q=59$

chute d'essai $H=15 \mathrm{~m}$

vitesse de rotation $n=600 \mathrm{t} / \mathrm{min}=10 \mathrm{~Hz}$

nombre aubes roue $Z_{r}=13$ (soient $n Z_{r}=1300 \mathrm{~Hz}$ nombre aubes

directrices

$$
Z_{D}=20\left\{\quad n Z_{R} Z_{D}=2600 \mathrm{~Hz}\right.
$$

2) La figure 1 présente l'emplacement du sonomètre Bruel et Kjaer 2203/1613 sur le modèle. Au voisinage immédiat du sonomètie est implanté un capteur de pression à membrane, à l'intérieur de la paroi plexiglas du cône de l'aspirateur.

3) A même chute d'essai, les mesures de bruit sont effectuées pour un régime de charge partielle typique, avec torche hélicoïdale bien établie, et pour un régime de forte charge. Dans ces deux types de fonctionnement, le coefficient de cavitation $\sigma$ est ajusté à cinq valeurs dégressives depuis 1,3 jusqu'à 0,05 .

Le tableau ci-après donne une vue d'ensemble des figures et du niveau sonore pondéré.

On peut observer immédiatement qu'en l'absence de figures de cavitation, les niveaux sonores à charge partielle et à forte charge sont très voisins, que ce niveau croit plus rapidement à charge partielle jusqu'au $\sigma=0,25$, mais qu'à partir du $\sigma=0,10$ le bruit à forte charge l'emporte très nettement comme conséquence directe d'une torche pulsatoire.

4) Les figures 2 et 3 montrent l'évolution du bruit par bandes de fréquences en fonction du $\sigma$ pour les deux régimes, et des remarques concluantes peuvent être faites: 


\begin{tabular}{|c|c|c|c|c|}
\hline \multicolumn{3}{|c|}{$\begin{array}{l}\text { REGIME DE CHARGE PARIIELLE } \\
\mathrm{a} / \mathrm{a} \text { opt }=0.64 \mathrm{H} / \mathrm{H} \text { opt }=1.0\end{array}$} & \multicolumn{2}{|c|}{$\begin{array}{l}\text { REGIME DE FORTE CHARGE } \\
\mathrm{a} / \mathrm{a} \text { opt }=1.3 \mathrm{H} / \mathrm{ll} \text { opt }=1.0\end{array}$} \\
\hline $\begin{array}{l}\text { Coefe. } \\
\text { Cavit. o }\end{array}$ & $\begin{array}{l}\text { Configuration visible et audible de } \\
\text { l'ecoulement }\end{array}$ & $\begin{array}{l}\text { Niveau sonore } \\
\text { pondéré (dBA) }\end{array}$ & $\begin{array}{l}\text { Configuration visible et andible de } \\
\text { l'ecoulentent }\end{array}$ & $\begin{array}{l}\text { Niveau sonore } \\
\text { pondérés (dBA) }\end{array}$ \\
\hline 1.2 & Aucune figure de cavilation & 77 & Aucune figure de cavitation & 78 \\
\hline 0.25 & $\begin{array}{l}\text { Vortex fins cavitant entre les aubes } \\
\text { ct débutant sur l'arete d'entree au } \\
\text { plafond. "lorche helicoüdale. Stable. }\end{array}$ & 82 & Torche axiale intermittente et courte & 79 \\
\hline 0.15 & $\begin{array}{l}\text { Les vortex s'allongent et dépassent } \\
\text { les aubes. } \\
\text { forche hélicoüdale donnant quelques } \\
\text { chocs dans le coude de laspirateur }\end{array}$ & 85 & $\begin{array}{l}\text { Torche axiale permanente disparais- } \\
\text { sant à mi-lauteur de l'aspirateur }\end{array}$ & 81 \\
\hline 0.10 & $\begin{array}{l}\text { Mémes Eigures de cavitation. } \\
\text { Cloos dans le coude aspirateur, ir- } \\
\text { réguliers et forts. }\end{array}$ & 85 & $\begin{array}{l}\text { 'lorche axiale jusqu'au fond du coude } \\
\text { aspirateur. Mouvement pulsatoire tres } \\
\text { net (variation de diametre du noyau à } \\
\text { environ } 2 \text { (Hz) }\end{array}$ & 90 \\
\hline 0.05 & $\begin{array}{l}\text { 1) isparition des chocs. La torche oc- } \\
\text { cupe la majeure section de l'aspira- } \\
\text { teur }\end{array}$ & 83 & $\begin{array}{l}\text { Ridedu de cavitation sur les parois } \\
\text { du cone de l'aspirateur, avec mouve- } \\
\text { ment pulsatoire du bas de ce rideau } \\
\text { chocs dans le coude. }\end{array}$ & 90 \\
\hline
\end{tabular}

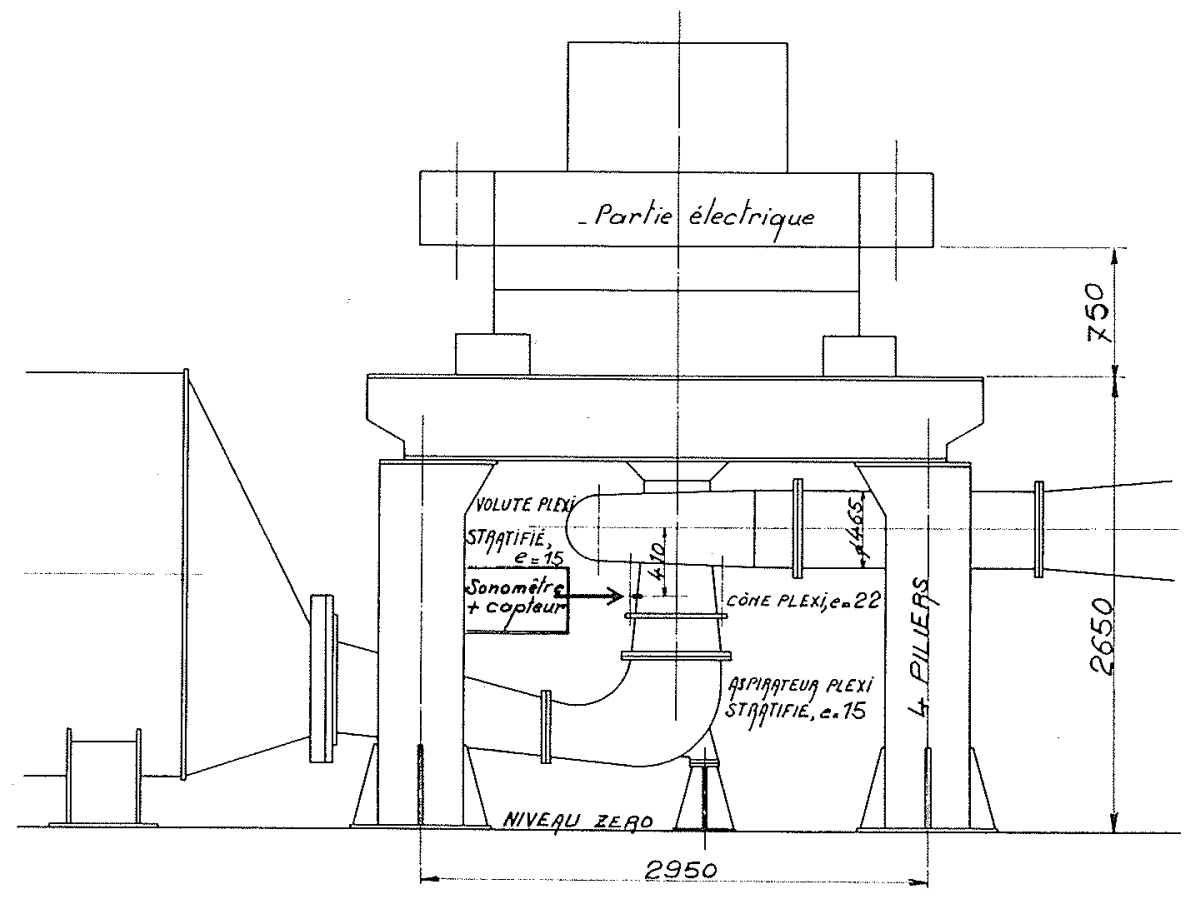

Figure 1. - Mesures de niveau sonore sur un modèle de turbine Francis. $\Phi$ Roue $=400 \mathrm{~mm}$.

Pompe alimentation à $-9,400$ m et 3 dalles partielles.

\section{A charge partielle (Fig. 2)}

L'apparition de la cavitation (vortex entre aubes + torche courte) a peu d'influence sur les fréquences inférieures à $1000 \mathrm{~Hz}$, mais entraine un accroissement sensible aux fréquences $\geqslant 2000 \mathrm{~Hz}$.

Le développement de la cavitation augmente les amplitudes des fréquences inférieures à $4000 \mathrm{~Hz}$, et les diminue au dessus de $4000 \mathrm{~Hz}$.

La généralisation de la cavitation $(\sigma<0,10)$ produit un effet inverse ; les basses fréquences voient leur amplitude diminuer très fortement au profit des hautes fré- quences. On note que le niveau sonore maximum est obtenu pour la bande des $250 \mathrm{~Hz}$ correspondant à peu près au premier harmonique du défilement des aubes.

\section{A forte charge (Fig. 3)}

L'apparition de la cavitation (torche axiale courte intermittente) produit peu d'effet à toutes fréquences.

Le développement de la cavitation (torche s'allongeant) ne donne pas de variation sonore aux basses fréquences, mais entraine un accroissement sensible aux hautes fréquences. 


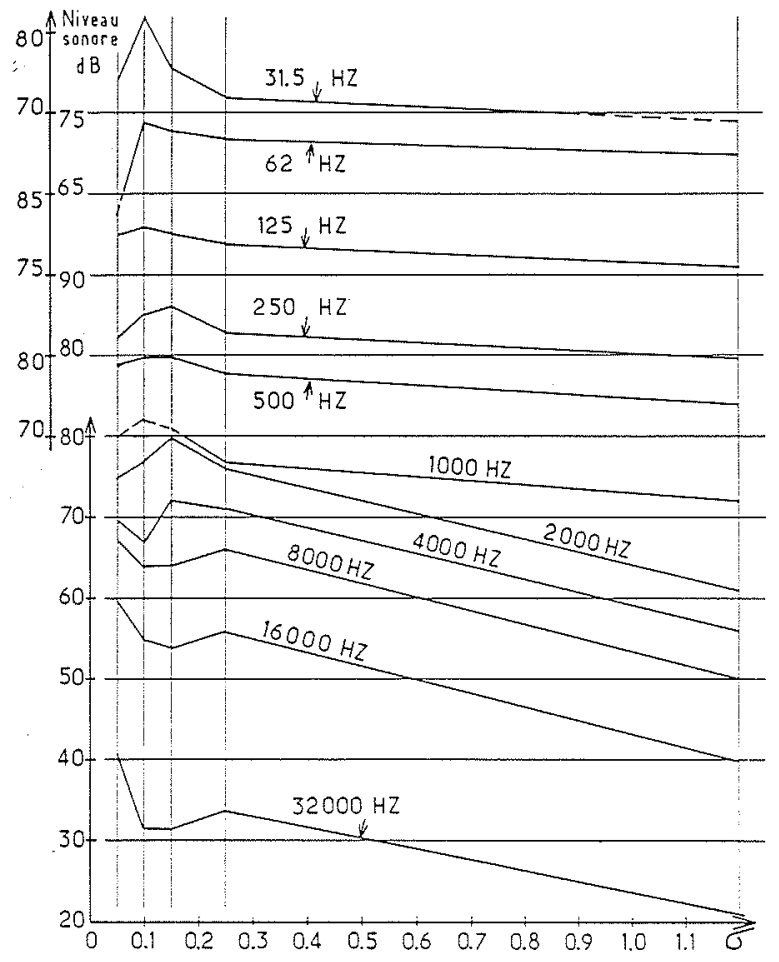

Figure 2. - Turbine Francis à charge partielle $(H$ essai $=15 \mathrm{~m})$. Influence de la cavitation sur le niveau sonore Mesures sur modèle réduit : a/a opt $=0,64 \mathrm{H} / \mathrm{H}$ opt $=1$.

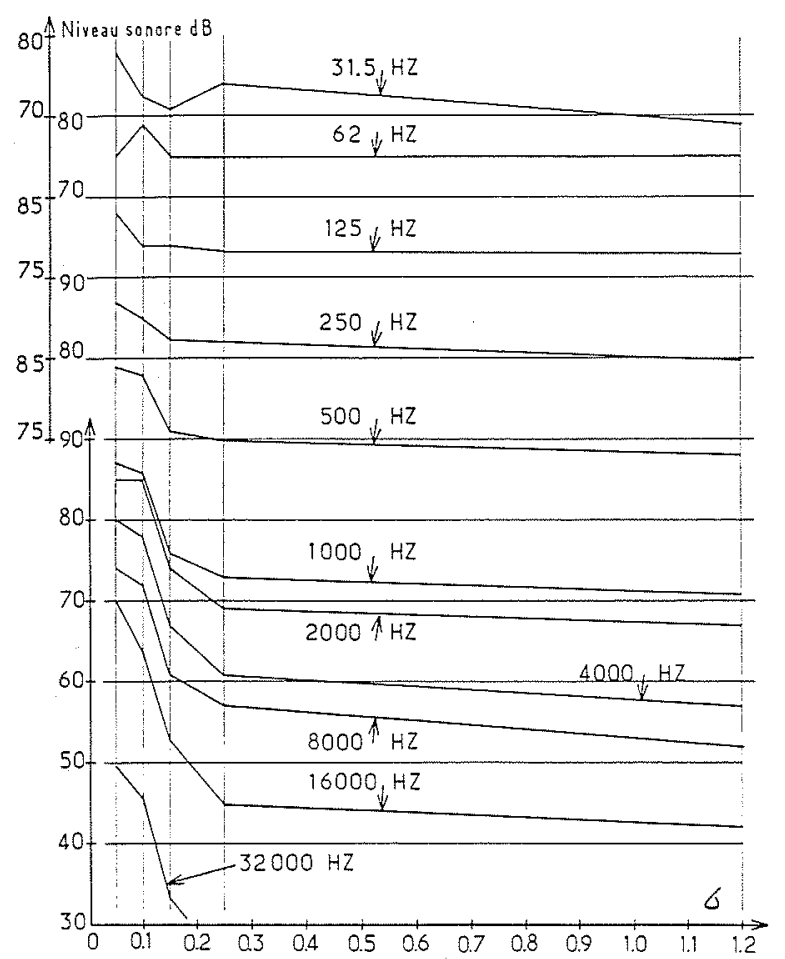

Figure 3. - Turbine Francis à forte charge ( $H$ essai $=15 \mathrm{~m}$ ). Influence de la cavitation sur le niveau sonore Mesures sur modèle réduit : $a / a$ opt $=1,3 \quad H / H$ opt $=1,0$.

Lorsque le noyau gazeux atteint le fond de l'aspirateur, une pulsation visible et audible commence. Un accroissement spectaculaire du niveau sonore des hautes fréquences se produit.

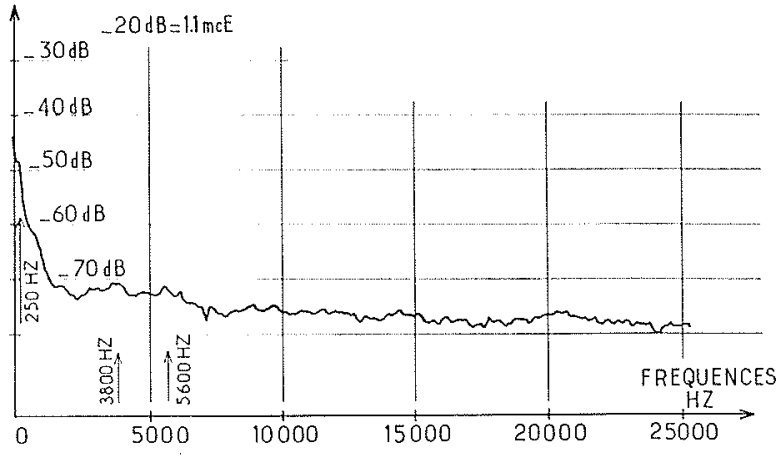

Figure 4. - Turbine Francis à forte charge $/ \sigma=0,10$. Pressions dynamiques en paroi du cone aspirateur.

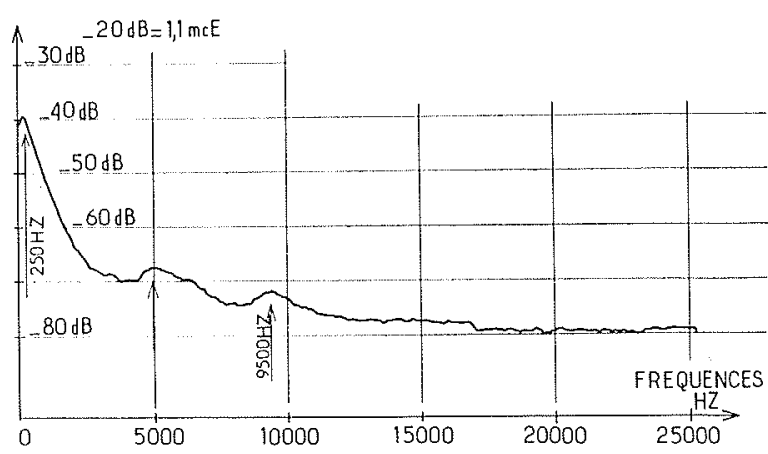

Figure 5. - Turbine Francis à forte charge $/ \sigma=0,05$. Pressions dynamiques en paroi du cone aspirateur.

Enfin, la généralisation de la cavitation entraine un accroissement général du niveau sonore, plus net cependant aux basses fréquences.

Les niveaux sonores maximum sont obtenus entre $250 \mathrm{~Hz}$ et $2000 \mathrm{~Hz}(85$ à $87 \mathrm{~dB})$.

5) En complément, les figures 4 et 5 donnent les spectres de fréquences des pressions hydrodynamiques mesurées en paroi du cône de l'aspirateur au voisinage immédiat du sonomètre, ceci pour le régime de charge partielle aux deux valeurs les plus basses du sigma.

On retrouve en tendance que l'excitation maximum se produit jusqu'au $1000 \mathrm{~Hz}$ et décroit rapidement au delà.

En revanche, lorsque $\sigma$ passe de 0,10 à 0,05 , le spectre des pressions s'amplifie nettement jusqu'à $5000 \mathrm{~Hz}$ et peu au delà. Le résultat sur les niveaux sonores était assez exactement inverse (Fig. 2). Cette apparente contradiction qui devra être expliquée se retrouve dans d'autres travaux [1].

\section{Niveaux sonores au-dessus d'une turbine maré- motrice réversible}

La figure 6 indique que le sonomètre est placé audessus de la fosse du groupe dans son axe.

La figure 7 synthétise les résultats obtenus en $\mathrm{dBC}$ pour les quatre quadrants du fonctionnement. En ce qui concerne les bruits minimum mesurés, le classement est 
donc le suivant :

Turbine directe

Pompe directe

$90 \mathrm{dBC}$

Turbine inverse

$94 \mathrm{dBC}$

Pompe inverse

$100 \mathrm{dBC}$

soit environ un doublement de l'intensité sonore à chaque changement de quadrant.

En ce qui concerne les bruits maximum mesurés, le classement devient :

$\begin{array}{ll}\text { Turbine directe } & 100 \mathrm{dBC} \\ \text { Pompe directe } & 102 \mathrm{dBC} \\ \text { Pompe inverse } & 105 \mathrm{dBC} \\ \text { Turbine inverse } & 106 \mathrm{dBC}\end{array}$

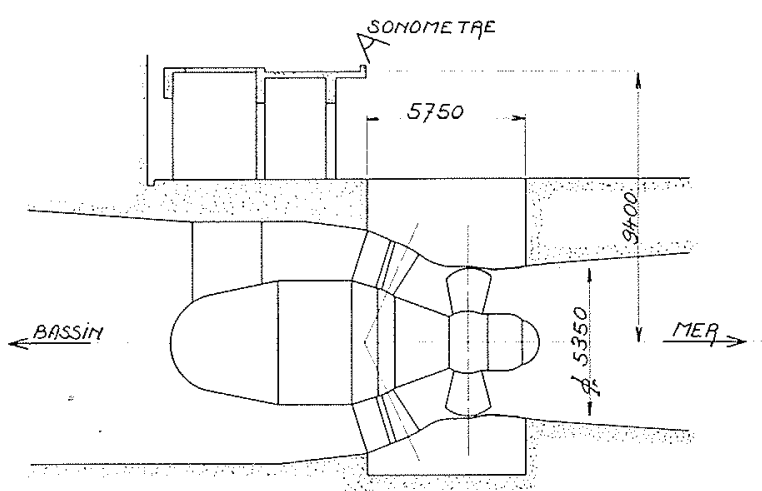

Figure 6. - Mesure de niveau sonore au dessus d'une turbine marémotrice réversible.

$H \approx 8 \mathrm{~m} \quad P \approx 10 \mathrm{MW}$.

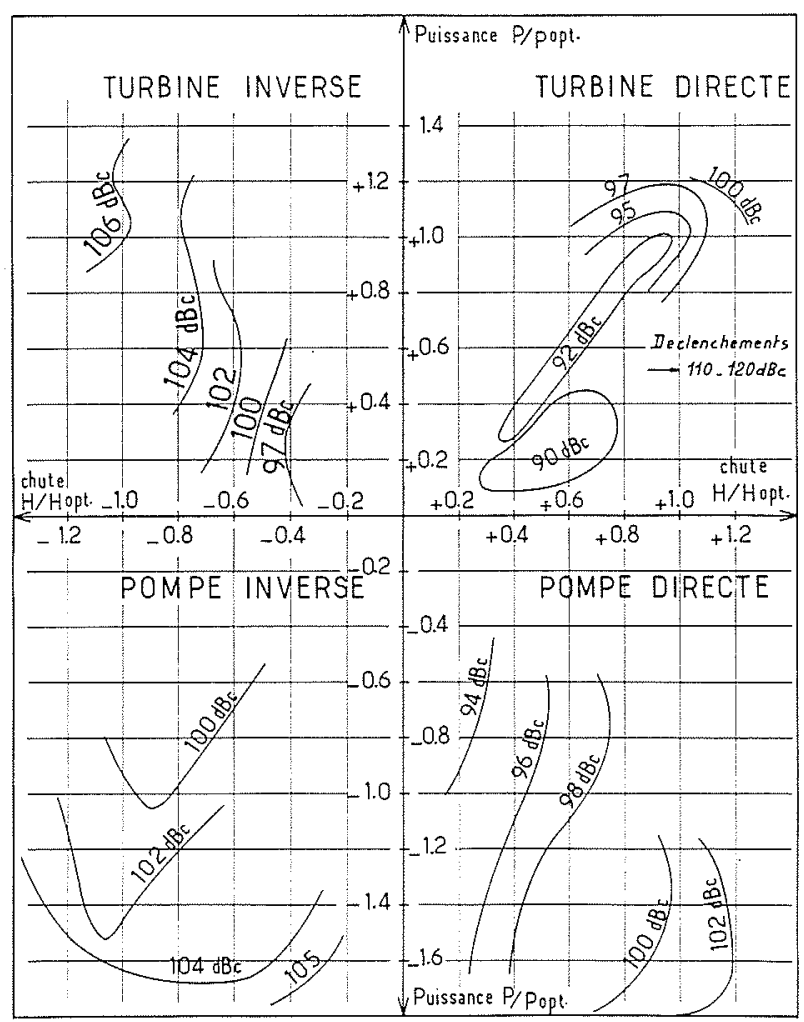

Figure 7. - Mesures de niveau sonore au dessus d'une turbine marémotrice réversible

$H \approx 8 \quad P \approx 10 \mathrm{MW}$
Sans entrer dans le détail, on peut dire que ces évolutions du niveau sonore sont assez logiques si l'on tient compte des réalités hydrodynamiques.

En turbine inverse, les aubes du rotor sont attaquées par leur bord le plus mince, donc le moins tolérant, tandis que les aubes directrices se trouvent à l'aval de l'écoulement du rotor, donc en cavitation et en attaques très irrégulières.

En pompe inverse, les aubes directrices se trouvent en dépression par rapport au rotor et cavitent considérablement, tandis que l'aspiration du rotor lui-même est perturbée.

Enfin, il peut être intéressant de rappeler comment se classent les rendements. Si celui de la turbine directe est pris égal à 1 , nous obtenons :

Rendement turbine directe

Rendement turbine inverse $\quad 0,84$

Rendement pompe directe $\quad 0,76$

Rendement pompe inverse $\quad 0,67$

\section{Niveaux sonores autour d'une turbine bulbe et d'une turbine Kaplan}

Les figures 8 et 9 présentent ces résultats et leur cornparaison est significative :

- à même localisation, le niveau sonore de la turbine Kaplan est très supérieur à celui du bulbe : ainsi, autour des pales, $111 \mathrm{dBA}$ contre 93,5 dBA; dans le puits turbine, $92 \mathrm{dBA}$ contre $87 \mathrm{dBA}$. Bien entendu, l'immersion de l'alternateur dans le cas du bulbe joue certainement un grand rôle ;

- de forts amortissements se produisent dès que l'on s'éloigne du puits turbine, mais là encore le bruit de l'alternateur devrait pouvoir être "séparé" de celui de la turbine.

\section{Niveaux sonores autour d'une turbine-pompe}

Dans une centrale comportant 4 groupes réversibles de type Francis ont été effectuées des mesures systéma-

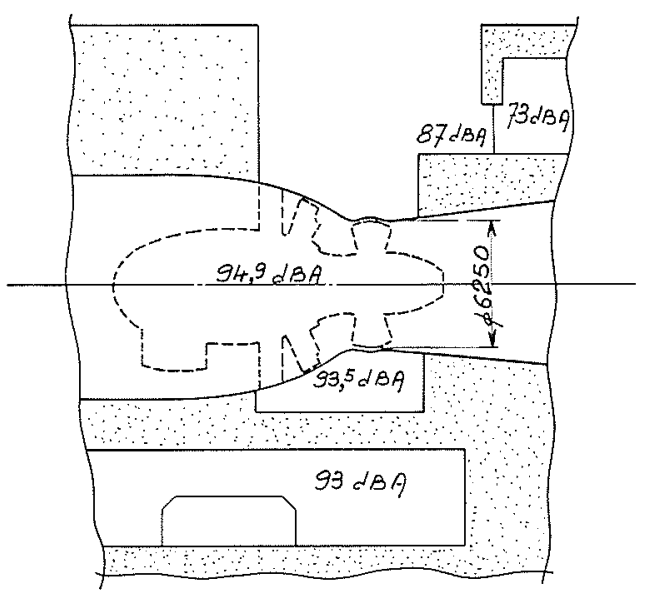

Figure 8. - Mesures d'ambiance sonore autour d'une turbine bulbe $H=4,93 \mathrm{~m} \quad P=10,9 \mathrm{MW}$ 


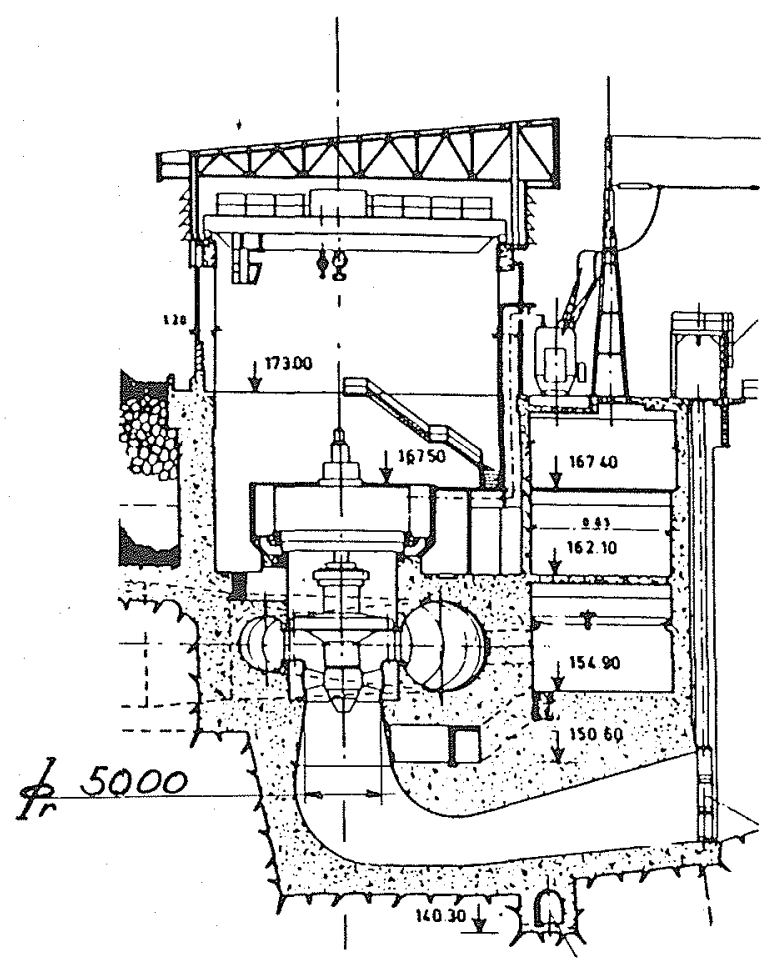

\begin{tabular}{|l|c|}
\hline \multicolumn{1}{|c|}{ Lieu de mesure } & $\mathrm{dBA}$ \\
\hline Salle auxiliaire électrique $162,1 \mathrm{~m}$ & 78 \\
\hline Puits turbine & 92 \\
\hline Auxiliaire $154,9 \mathrm{~m}$ & 95 \\
\hline Couloir aspirateur $151,8 \mathrm{~m}$ & 100 \\
\hline Porte aspirateur $151,8 \mathrm{~m}$ & 102 \\
\hline Pourtour ceinture & 111 \\
\hline Salle des commandes $167,4 \mathrm{~m}$ & 72 \\
\hline Bureau amont $167,4 \mathrm{~m}$ & 75 \\
\hline Bureau aval $167,4 \mathrm{~m}$ & 72,5 \\
\hline Salle des machines $167,4 \mathrm{~m}$ & 81 \\
\hline
\end{tabular}

Figure 9. - Mesure d'ambiance sonore autour d'une turbine Kaplan. $H=26,85 \mathrm{~m} \quad P=53 \mathrm{MW}$.

tiques au sonomètre, soit par bande de fréquences, soit par pondération.

Chaque groupe présente les caractéristiques suivantes:

$H \max =400 \mathrm{~m}$

Vitesse $=600 \mathrm{rpm}=10 \mathrm{~Hz}$

Puissance $=135 \mathrm{MW}$

Nombre d'aubes du rotor $Z_{R}=9$ du distributeur $Z_{D}=16$

Les figures 10,11 et le tableau II présentent les principaux résultats, qui appellent les remarques suivantes :

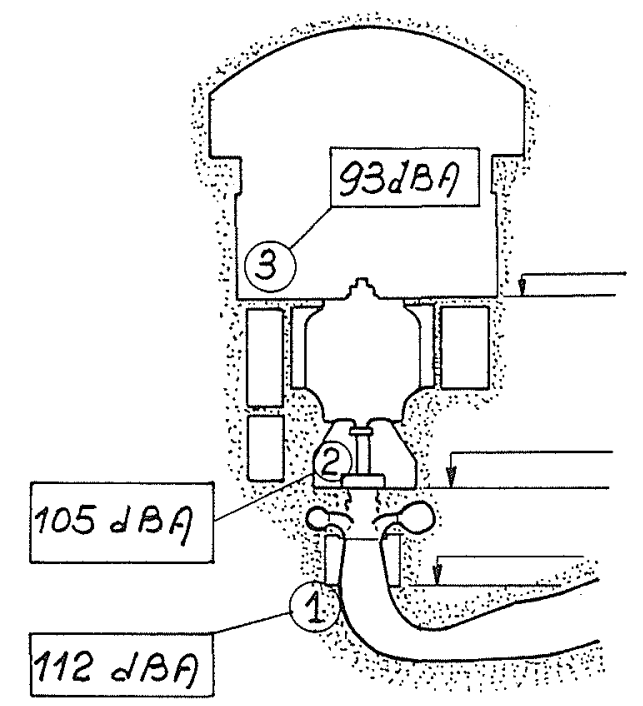

Figure 10. - Niveaux sonores en trois sites d'une turbine pompe fonctionnant en turbine.
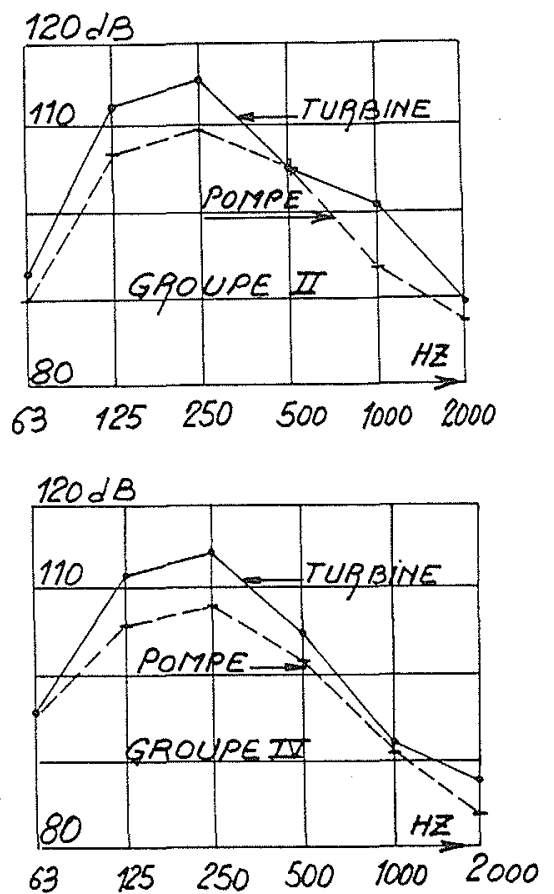

Figure 11. - Niveaux sonores dans la salle des turbines en fonction de la fréquence.

- les niveaux de bruit maximum sont très importants, même en régime nominal de turbine. Cependant, si on les compare à ceux de la turbine Kaplan déjà examinée, on ne peut dire que l'accroissement soit excessif, puisqu'avec une chute passant de $25 \mathrm{~m}$ à $400 \mathrm{~m}$, on passe en paroi de l'aspirateur d'un niveau sonore $102 \mathrm{dBA}$ à un niveau $112 \mathrm{dBA}$. On notera (tableau II) que dans la salle des turbines le niveau de bruit lors du démarrage en pompe atteint $122 \mathrm{dBA}$. A ce degré, le danger humain est évident, ce n'est plus seulement une gêne; 
Tableau II. - Niveaux sonores comparés dans la salle des machines

\begin{tabular}{|c|c|c|c|c|c|}
\hline \multirow{2}{*}{ Régime } & \multirow{2}{*}{ Groupe } & \multicolumn{4}{|c|}{ Niveaux sonores } \\
\hline & & A & B & C & LIN \\
\hline Pompe & $\begin{array}{c}\text { I } \\
\text { II } \\
\text { III } \\
\text { IV }\end{array}$ & $\begin{array}{c}99 \\
100 \\
- \\
99\end{array}$ & $\begin{array}{c}104 \\
106,5 \\
\overline{-} \\
105\end{array}$ & $\begin{array}{r}106 \\
108 \\
- \\
107\end{array}$ & $\begin{array}{c}106,5 \\
108 \\
-\end{array}$ \\
\hline $\begin{array}{c}\text { Démarrage } \\
\text { Pompe }\end{array}$ & & 122 & & & \\
\hline $\begin{array}{l}\text { Arrêt } 4 \\
\text { Groupes }\end{array}$ & & \multicolumn{4}{|c|}{ Restent $86 \mathrm{~dB}$ entre 500 et $2000 \mathrm{HZ}$} \\
\hline Turbine & $\begin{array}{c}1 \\
11 \\
111 \\
\text { IV }\end{array}$ & $\begin{array}{l}100 \\
109 \\
100 \\
101\end{array}$ & $\begin{array}{l}107 \\
108 \\
106,5 \\
108,5\end{array}$ & $\begin{array}{l}108,5 \\
110 \\
108 \\
110,5\end{array}$ & $\begin{array}{l}109 \\
110 \\
108 \\
110,5\end{array}$ \\
\hline
\end{tabular}

- le régime pompe est plutôt moins bruyant que le régime turbine, mais dans les deux cas la bande de fréquence privilégiée est celle des $250 \mathrm{~Hz}$, c'est-à-dire voisine du premier harmonique du défilement des aubes. Ce résultat avait déjà été constaté lors des mesures sur modèle réduit de turbine Francis ;

- le niveau résiduel pendant l'arrêt des 4 groupes est encore de $86 \mathrm{~dB}$ émis par les auxiliaires.

\section{Quelques conclusions}

1) Les relations qualitatives constatées entre les niveaux sonores et les phénomènes hydrodynamiques paraissent conformes à la logique et recoupent assez bien les exemples de la littérature $[1,2,3,4$ et autres].

2) En ce qui concerne la cavitation, les mesures faites sur modèle réduit montrent bien les deux types de bruit qu'elle engendre, à savoir :

- d'autre part, un bruit de basses fréquences correspondant aux variations de volume en masse des noyaux de vapeur (torche);

- d'autre part, un bruit de hautes fréquences correspondant à l'implosion des "familles" de microbulles.
Dans chacun des cas, le niveau sonore est d'autant plus important que le gradient de pression est raide, qu'il provienne d'une caractéristique géométrique (coude de l'aspirateur) ou d'une caractéristique de régime (pulsation d'une torche axiale). L'intensité maximum maximorum sera atteinte pour des implosions totales d'une grande masse de vapeur, comme c'est le cas, heureusement rare, de certains régimes de charge partielle dans des aspirateurs mal conçus ou trop peu profonds.

3) Le respect des cahiers des charges en matière de bruit pose des problèmes très sérieux:

- le lieu de mesure doit être évidemment normalisé en fonction du but recherché ;

- le bruit mesuré devrait être celui de la machine hydraulique, indépendamment des bruits parasites et des bruits réfléchis, sachant que la machine électrique constitue alors un bruit "parasite". En effet, si les différentes sources ne sont pas cernées et différenciées, on voit mal comment répartir les responsabilités entre hydraulique, génération électrique, génie civil et architecture, auxiliaires... On peut rappeler par exemple qu'une source sonore peut voir son niveau multiplié par 8 , soit $9 \mathrm{~dB}$, si elle se trouve localisée à la jonction de trois plans réfléchissants;

- le problème scientifique et technique à résoudre est donc de déterminer l'intensité acoustique vraie d'une source, c'est-à-dire son intensité vectorielle et non scalaire. Des méthodes existent [5]; il reste à organiser leur application à des machines aussi volumineuses et peu accessibles que les turbomachines hydroélectriques.

\section{Bibliographie}

[1] TOURRET J. - Problèmes associés à l'étude du bruit d'un circuit de pompes centrifuges. La Houille Blanche $n^{\circ} 2 / 3-$ 1979.

[2] RATA M. - Recensement des méthodes d'observation de la cavitation par voie acoustique. La Houille Blanche $\mathrm{n}^{\circ} 6-$ 1963.

[3] VARGA J. et SEBESTYEN G. - Experimental investigation of cavitation noise. La Houille Blanche $\mathrm{n}^{\circ} 8-1966$.

[4] FLORJANCIC D., SCHUFFLER W., ZOGG H. - Réduction primaire du bruit dans les pompes centrifuges. Revue SULZER $1 / 1980$.

[5] TOURRET J. - La mesure du bruit émis par les réducteurs, nouvelles techniques. Note CETIM. 